MITCTP\#2326

UBCTP-94-004

July, 1994

\title{
CANONICAL BF-TYPE TOPOLOGICAL FIELD THEORY AND FRACTIONAL STATISTICS OF STRINGS
}

\author{
Mario Bergeron*,** \\ Center for Theoretical Physics, Laboratory for Nuclear Science and Department of Physics \\ Massachusetts Institute of Technology, Cambridge, Massachusetts, 02139 U. S. A. \\ Gordon W. Semenoff* and Richard J. Szabo* \\ Department of Physics, University of British Columbia \\ Vancouver, British Columbia, V6T 1 Z1 Canada
}

Submitted to Nuclear Physics B

\begin{abstract}
We consider BF-type topological field theory coupled to non-dynamical particle and string sources on spacetime manifolds of the form $\mathbb{R}^{1} \times \mathcal{M}_{3}$, where $\mathcal{M}_{3}$ is a 3-manifold without boundary. Canonical quantization of the theory is carried out in the Hamiltonian formalism and explicit solutions of the Schrödinger equation are obtained. We show that the Hilbert space is finite dimensional and the physical states carry a one-dimensional projective representation of the local gauge symmetries. When $\mathcal{M}_{3}$ is homologically nontrivial the wavefunctions in addition carry a multi-dimensional projective representation, in terms of the linking matrix of the homology cycles of $\mathcal{M}_{3}$, of the discrete group of large gauge transformations. The wavefunctions also carry a one-dimensional representation of the non-trivial linking of the particle trajectories and string surfaces in $\mathcal{M}_{3}$. This topological field theory therefore provides a phenomenological generalization of anyons to $(3+1)$ dimensions where the holonomies representing fractional statistics arise from the adiabatic transport of particles around strings. We also discuss a duality between large gauge transformations and these linking operations around the homology cycles of $\mathcal{M}_{3}$, and show that this canonical quantum field theory provides novel quantum representations of the cohomology of $\mathcal{M}_{3}$ and its associated motion group.
\end{abstract}

\footnotetext{
* Work supported in part by the Natural Sciences and Engineering Research Council of Canada.

** Work supported in part by funds provided by the U.S. Department of Energy (D.E.O.) under the contract \#DE-AC02-76ER03069 and the cooperative agreement \#DE-FC02-94ER40818.
} 


\section{INTRODUCTION}

In $(2+1)$ dimensional spacetime, anyons have a by now well-known physical realization where magnetic flux tubes are attached to charged particles and the Aharonov-Bohm phases resulting from adiabatic transport of the composites give them fractional exchange statistics (see [1] for a review). Anyons are also known to be phenomenologically described in quantum field theory by Chern-Simons gauge theory, a topological field theory [1,2] . We would expect these fractional phases to survive in a 4 dimensional world where flux tubes have infinite extent, as in the conventional Aharanov-Bohm effect. In this Paper we shall study a particular topological quantum field theory which gives the appropriate generalization of anyons to $(3+1)$ dimensions.

Holonomy effects in physical systems should always be described by some topological field theory. In the following we shall consider a certain class of Schwarz-type topological gauge theories, the so-called BF-theories [2-5], which describe the type of holonomy which can occur in adiabatic transport in a theory of point charges and strings where all other degrees of freedom decouple. Abelian BF theories in 4 dimensions have for a long time been known to be especially suited to describe physical systems where the sources are vortex-like configurations, and it is well-known that there is a duality between dynamical BF-type gauge theories and theories involving Nambu-Goldstone fields with global abelian symmetries. This fact has been used to describe an alternative approach to the Higgs mechanism, and it leads to the London constitutive equation in superconductivity [6]. It has also recently been exploited in dual models of cosmic strings [7], and axionic black hole theories [8] where the axion charge is physically detectable only by external cosmic strings in a 4 dimensional Aharanov-Bohm type process [9] identical to what we shall consider here. These 4 dimensional Aharanov-Bohm phases also appear in field theories of the QCD string [10].

On the mathematical side, it was realized in the late 1970's by Schwarz that both abelian and nonabelian BF-theories in $d$ dimensions give effectively computable path integral representations of a particular topological invariant of $d$-manifolds, the Ray-Singer analytic torsion $[2,3,5,11]$. The properties of this non-trivial topological invariant thus have natural interpretations through standard techniques of quantum field theory. Furthermore, just as Chern-Simons theory gives representations of linking numbers of curves

in 3 dimensions [2], BF theories provide path integral representations of the linking and 
intersection numbers of generic surfaces in $d$ dimensions $[2,3,5]$.

Not unrelated to these properties of BF theory is the fact that the statistical phases which arise in some models of heterotic string compactification illustrate explicitly the possibility of unusual statistics in 4 dimensional string theory [12]. These statistical phases can be seen to arise from certain cosmic string and superstring phenomena, such as anomaly cancellation in 4 dimensions [13], and they arise from BF-type theories of superstrings such as Nambu-Goto string theory modified with the inclusion of the Kalb-Ramond term [14]. This possibility was made explicit by Aneziris et al. who showed that more general statistics can exist for strings in $(3+1)$ dimensions [15]. Just as for particles moving on a plane where the configuration space monodromies lead one to consider the braid group of the plane, whose representations allow for exotic statistics of charged particles in $(2+1)$ dimensions [1], Aneziris et al. constructed representations of the holonomies of closed paths in the configuration space of a system of identical, oriented strings which demonstrate the possibility of fractional statistics.

The idea of fractional statistics for a system of identical strings requires a better understanding of the Hilbert space of physical states for such a system [12]. In this Paper we shall consider $(3+1)$ dimensional abelian BF-type topological field theory coupled to non-dynamical string sources, but in the limiting case where one set of strings can be considered as points in space (the situation in cosmic string scenarios) so that the composite states are point particles with infinitely thin flux tubes attached (Section 2), the same situation that arises in pure Chern-Simons gauge theory. One advantage of this simplifying limit is that we need not worry about regulating the usual divergences which arise from self-linking terms. We shall also consider only spacetime manifolds without boundary ${ }^{1}$.

We explicitly construct the Hilbert space of the theory by considering the canonical quantization of this model in the Hamiltonian formalism. We begin by examining how particle-string holonomies are represented in the wavefunctions when the spatial 3-manifold is flat Euclidean 3 -space $\mathbb{R}^{3}$ (Section 3 ). There we obtain a simple representation of the particle-string linking in $\mathbb{R}^{3}$ through a spherical solid angle function which is the 3 dimensional version of the usual multivalued angle function which appears in planar anyon theories [1]. In this case the Hilbert space is one dimensional and the physical states carry a unitary representation of both the statistics and the local gauge group.

For spacetime manifolds with boundary, the analogs of the boundary localized edge states and vertex operators of Chern-Simons theory have been studied in [16]. 
We then consider the case where the spatial manifold is curved, and also allow for non-trivial spatial topology (Section 4). There we obtain a generalization of the adiabatic particle-string linking found in Euclidean space through a generalized curved space spherical angle function, and the wavefunctions again carry a one dimensional unitary representation of the fractional statistics and of the local gauge symmetries. When the space has non-trivial homology, we find that it is necessary to normalize the cohomological parts of the gauge fields in terms of the linking matrix of the homology cycles [17] in order for the correct particle-string linking representation to appear. We shall also see that, when the coefficient of the BF action (the "statistics parameter") is of the form $M k_{1} / k_{2}$ with $k_{1}$ and $k_{2}$ integers and $M$ the integer-valued determinant of the linking matrix of the $p$ homology 1-cycles with the $p$ homology 2-cycles of the 3-manifold, the Hilbert space is $\left|k_{1} k_{2} M\right|^{p}$ dimensional and the physical states in addition carry a $\left|k_{2}\right|^{p}$ dimensional projective representation of the associated algebra of large gauge transformations. This algebra and the ensuing representations are determined by the linking matrix of the space, and thus the BF theory leads to a novel, complete quantum representation of its homological properties. We shall further show that these large gauge transformations are dual to the linkings of particles and strings around the non-trivial homology cycles of the manifold, and their representation within the physical states provides a $\left|k_{1}\right|^{p}$ dimensional projective representation of the first and second cohomology of the underlying space. The wavefunctions thus also yield intriguing representations of the fundamental group of the particle-string configuration space.

These results shed light on the structure of the enlarged Hilbert space for 4 dimensional strings induced by the more complicated fundamental group of the string configuration space. They also illustrate the complexity of the quantum theory of anyonic systems in (3 +1 ) dimensions, and show as well the rich mathematical structure inherent in BF theory insofar as providing topological invariants in 3 dimensions. Some details of the calculations in the case of a curved manifold are summarized in an Appendix at the end of the Paper, where we derive an explicit expression for the generalized adiabatic linking function. 


\section{CANONICAL BF THEORY IN FOUR DIMENSIONS}

We begin by describing some of the general features of 4-dimensional BF-type topological field theory and its canonical formalism when coupled to sources. Consider the field theory of a real-valued 2-form field $B$ and a real-valued 1-form field $A$ defined on a 4 dimensional spacetime manifold $\mathcal{M}_{4}$ with metric of Minkowski signature. The $\mathrm{BF}$ action is given by the spacetime integral of a 4 -form $[2-5]$

$$
S=\int_{\mathcal{M}_{4}} \frac{k}{2 \pi} B \wedge d A
$$

where $\frac{1}{k}$ is the coupling constant. This action is invariant under the gauge transform

$$
A \rightarrow A+\chi
$$

where $\chi$ is a closed 1-form, $d \chi=0$. Modulo elements of the first cohomology group of $\mathcal{M}_{4}$ this is trivially satisfied by an exact 1-form $\chi=d \chi^{\prime}$. On the other hand, like Chern-Simons theory in $(2+1)$ dimensions [2] the action transforms by a surface term under

$$
B \rightarrow B+\xi
$$

where $\xi$ is a closed 2-form, $d \xi=0$, which is also satisfied by an exact 2-form $\xi=d \xi^{\prime}$ modulo elements of the second cohomology group of $\mathcal{M}_{4}$.

In the present field theory without sources any closed forms are allowed in (2.2) and (2.3). However, when this topological field theory is coupled to sources we also require gauge invariance of the Wilson operators

$$
W[L]=\exp \left(i \int_{L} A\right) \quad, \quad W[\Sigma]=\exp \left(i \int_{\Sigma} B\right)
$$

for any oriented loop $L$ and any compact orientable surface $\Sigma$ in $\mathcal{M}_{4}$. This restricts the cohomological parts of the closed forms allowed in (2.2) and (2.3) to those with integervalued cohomology so that

$$
\int_{L} \chi=2 \pi n \quad, \quad \int_{\Sigma} \xi=2 \pi m
$$

for some integers $n$ and $m$. In the following we shall assume this restricted gauge symmetry.

The partition function is given by the path integral

$$
Z=\int D A D B \exp \left(i \int_{\mathcal{M}_{4}} \frac{k}{2 \pi} B \wedge d A\right)
$$


The functional integrals in (2.6) are performed over all gauge orbits and are normalized by the volume of the gauge group. This path integral is related to the Ray-Singer analytic torsion [18] which is a topological invariant of $\mathcal{M}_{4}$ given by properties of the spectrum of the differential operators $d$ and $\star d$ and the Laplacian $\square_{n}$ acting on $n$-forms on $\mathcal{M}_{4}$, and here is given explicitly by the ratio of determinants $[2,3,11]$

$$
Z=\frac{\operatorname{det}_{\perp}^{1 / 2} \square_{1}}{\operatorname{det}_{\perp}^{1 / 2} \square_{0} \operatorname{det}_{\perp}^{1 / 4} \square_{2}}
$$

where $\operatorname{det}_{\perp}$ denotes the determinant with zero modes arising from gauge invariance excluded.

Gauge- and topologically-invariant operators in this quantum field theory are given by 2-cycle holonomies of $B$ and 1-cycle holonomies of $A$. The expectation value of the Wilson loop and surface variables (2.4) is given by the path integral with sources

$$
<W[L], W[\Sigma]>=\frac{\int D A D B \exp \left(i \int_{\mathcal{M}_{4}} \frac{k}{2 \pi} B \wedge d A+i \int_{L} A+i \int_{\Sigma} B\right)}{\int D A D B \exp \left(i \int_{\mathcal{M}_{4}} \frac{k}{2 \pi} B \wedge d A\right)}
$$

where the Wilson loops are the world-lines $L$ of particles and surfaces are the world-sheets $\Sigma$ of strings. This integral is independent of the metric of $\mathcal{M}_{4}$ and is also formally a topological invariant. It is related to the topological linking number of surfaces $\Sigma$ with contours $L$. This can be seen by fixing the gauge and performing the integral to obtain $[2,3,5]$

$$
<W[L], W[\Sigma]>=\exp \left(-\frac{2 i}{\pi k} \int_{\Sigma} d \Sigma_{\mu \nu}(x) \int_{L} d l_{\sigma}(y) \epsilon^{\mu \nu \sigma \rho} \frac{(x-y)_{\rho}}{|x-y|^{4}}\right)=\mathrm{e}^{-\frac{2 \pi i}{k} I(\Sigma, L)}
$$

where $d \Sigma_{\mu \nu}(x)=d^{2} \sigma \epsilon^{\alpha \beta} \frac{\partial x_{\mu}}{\partial \sigma^{\alpha}} \frac{\partial x_{\nu}}{\partial \sigma^{\beta}}$ is the differential string area element and $l_{\sigma}$ parametrizes the particle world-line. Here we have for simplicity assumed that $\mathcal{M}_{4}=\mathbb{R}^{1} \times \mathbb{R}^{3}$ and we use the convention $\epsilon^{0123}=+1$. (2.9) yields the standard expression for the Gaussian linking number $I(\Sigma, L)$ of a contour $L$ and a surface $\Sigma$ [19]. Path integral representations of these linking numbers in arbitrary 4-manifolds can also be obtained $[2,3,5]$ (see the Appendix, equation (A.23)).

A more complete picture of this particle-string system is obtained by canonical quantization. For this we choose the spacetime to be the product manifold $\mathbb{R}^{1} \times \mathcal{M}_{3}$ where $\mathbb{R}^{1}$ 
parametrizes the time. The action is now ${ }^{2}$

$$
S=\int d t \int_{\mathcal{M}_{3}} d^{3} x\left(\frac{k}{4 \pi} \epsilon^{\mu \nu \rho \sigma} B_{\mu \nu} \partial_{\rho} A_{\sigma}+A_{\mu} j^{\mu}+\frac{1}{2} B_{\mu \nu} \Sigma^{\mu \nu}\right)
$$

where the particle current is given by

$$
j^{\mu}(x)=\sum_{a} q_{a} \int_{L_{a}} d \tau \frac{d r_{a}^{\mu}(\tau)}{d \tau} \delta^{(4)}\left(x, r_{a}(\tau)\right)
$$

with $r_{a}^{\mu}(\tau)$ the embedding of the world line $L_{a}$ of particle $a$ with charge $q_{a}$ in $\mathcal{M}_{4}$. It satisfies the continuity equation $\partial_{\mu} j^{\mu}=0$ when the world lines are closed. The string current is given by

$$
\Sigma^{\mu \nu}(x)=\sum_{b} \phi_{b} \int_{\Sigma_{b}} d^{2} \sigma \epsilon^{\alpha \beta} \frac{\partial X_{b}^{\mu}(\sigma)}{\partial \sigma^{\alpha}} \frac{\partial X_{b}^{\nu}(\sigma)}{\partial \sigma^{\beta}} \delta^{(4)}\left(x, X_{b}(\sigma)\right)
$$

with $X_{b}^{\mu}(\sigma)$ the embedding of the world-sheet $\Sigma_{b}$ of string $b$ with "electromagnetic" flux $\phi_{b}$ in $\mathcal{M}_{4}$. It is antisymmetric, $\Sigma^{\mu \nu}=-\Sigma^{\nu \mu}$, and conserved, $\partial_{\mu} \Sigma^{\mu \nu}=0$, when either the string world-sheet is closed or the embedding obeys the appropriate boundary conditions.

When the 3-manifold $\mathcal{M}_{3}$ is compact, we require that these sources further obey the property that the total charge and total flux both vanish, $\sum_{a} q_{a}=\sum_{b} \phi_{b}=0$. The gauge constraints (see (2.13) below) imply that the inclusion of non-zero charge and flux sectors of the theory require the gauge connections $A$ and $B$ to be sections of some vector bundles over $\mathcal{M}_{3}$ (as then they have non-vanishing curvature), and they can only be defined locally on patches over $\mathcal{M}_{3}$. In this case the BF action (2.1) must be modified appropriately [20], and we shall not consider this technical adjustment in this Paper. Intuitively, the constraint that the total charge and flux vanish in a closed space is required since their electric and "magnetic" fluxes have nowhere to go.

The temporal components of the fields are Lagrange multipliers which enforce the constraints

$$
\frac{k}{2 \pi} \partial_{i} B^{i}+j^{0} \sim 0 \quad, \quad \frac{k}{2 \pi} \epsilon^{i j k} \partial_{j} A_{k}+\Sigma^{0 i} \sim 0
$$

where $B^{i}(x)=\frac{1}{2} \epsilon^{i j k} B_{j k}(x)$. The second constraint in (2.13) confines "electromagnetic" flux to the string world sheets and gives the analog of the Meissner effect in a BCS superconductor [6]. The first constraint then couples this flux to the particle charges with

In this Paper we shall implicitly assume that all antisymmetric tensors, Dirac delta functions, spatial index contractions and volume forms $d^{3} x$ contain the appropriate metric factors required for diffeomorphism invariance. Also, in the following $*$ denotes the Hodge duality operator defined with respect to the metric of $\mathcal{M}_{3}$. 
coupling $\frac{1}{k^{2}}$. Thus the propagating gauge degrees of freedom in this field theory decouple and their only roles are to attach infinitely long flux tubes to the point charges in this theory in terms of the coefficient $k$. As in Chern-Simons theory [1], it is this coupling which leads to Aharanov-Bohm phases which are ultimately responsible for the statistics of these charged particle composites.

The remaining action

$$
S=\int d t \int_{\mathcal{M}_{3}} d^{3} x\left(\frac{k}{2 \pi} B^{i} \dot{A}_{i}+A_{i} j^{i}+\frac{1}{2} B_{i j} \Sigma^{i j}\right)
$$

is of first order in time derivatives and is therefore already expressed in phase space with the spatial components of $A$ and $B$ being the canonically conjugate variables. It leads to the canonical commutator

$$
\left[A_{i}(x), B^{j}(y)\right]=\frac{2 \pi i}{k} \delta_{i}^{j} \delta^{(3)}(x, y)
$$

In the temporal gauge $A_{0}=0, B_{0 i}=0$, the Hamiltonian is

$$
H=\int_{\mathcal{M}_{3}} d^{3} x\left(-A_{i} j^{i}-\frac{1}{2} B_{i j} \Sigma^{i j}\right)
$$

The canonical formalism above can now be used to solve for the spectrum of the Hamiltonian (2.16). In the following we shall show that the associated Hilbert space states represent fractional particle-string linking numbers as well as the possible non-trivial topological properties of the 3 -manifold $\mathcal{M}_{3}$.

\section{PARTICLE-STRING HOLONOMY IN EUCLIDEAN SPACE}

To get an intuitive idea of how the wavefunctions of BF-theory represent fractional linking numbers of particles and strings, we first consider the relatively simple case where $\mathcal{M}_{3}$ is Euclidean 3-space $\mathbb{R}^{3}$. We can decompose the fields over $\mathbb{R}^{3}$ using the Hodge decomposition, assuming that they have compact support. We then have the usual longitudinal and transverse decompositions

$$
\begin{gathered}
A=d \theta+* d K^{\prime} \quad \text { or } \quad A_{i}=\nabla_{i} \theta+\epsilon_{i j k} \nabla^{j} K^{\prime k} \\
* B=d \theta^{\prime}+* d K \quad \text { or } \quad B^{i}=\nabla^{i} \theta^{\prime}+\epsilon^{i j k} \nabla_{j} K_{k}
\end{gathered}
$$


The commutation relations (2.15) now take the form

$$
\left[\theta(x), \theta^{\prime}(y)\right]=-\frac{2 \pi i}{k} \frac{1}{\nabla^{2}} \delta^{(3)}(x, y) \quad, \quad\left[K_{i}(x), K^{\prime j}(y)\right]=\frac{2 \pi i}{k}\left(\delta_{i}^{j}-\frac{\nabla_{i} \nabla^{j}}{\nabla^{2}}\right) \frac{1}{\nabla^{2}} \delta^{(3)}(x, y)
$$

and can be represented by the functional derivative operators

$$
\begin{aligned}
& A_{i}(x)=\nabla_{i} \theta(x)-\frac{2 \pi i}{k} \epsilon_{i j k} \nabla^{j} \frac{1}{\nabla^{2}} \frac{\delta}{\delta K_{k}(x)} \\
& B^{i}(x)=\epsilon^{i j k} \nabla_{j} K_{k}(x)+\frac{2 \pi i}{k} \nabla^{i} \frac{1}{\nabla^{2}} \frac{\delta}{\delta \theta(x)}
\end{aligned}
$$

We treat the constraints (2.13) as physical state conditions which separate the gaugeinvariant states in the Hilbert space of the canonical quantum field theory. In the functional Schrödinger picture, we see from (3.4) that they are solved by wavefunctionals of the form

$$
\Psi_{\text {phys }}[\theta, K ; t]=\exp \left[i \int d^{3} x\left(\theta(x) j^{0}(x, t)+K_{i}(x) \Sigma^{0 i}(x, t)\right)\right] \tilde{\Psi}(t)
$$

Note that the continuity equation $\partial_{\mu} \Sigma^{\mu 0}=0$ is required for the constraints (2.13) to be satisfied in (3.5) here. The time-independent gauge symmetries (2.2) and (2.3) are therefore represented projectively as

$$
\Psi_{\text {phys }}\left[\theta+\chi^{\prime}, K+\xi^{\prime} ; t\right]=\exp \left[i \int d^{3} x\left(\chi^{\prime}(x) j^{0}(x, t)+\xi_{i}^{\prime}(x) \Sigma^{0 i}(x, t)\right)\right] \Psi_{\text {phys }}[\theta, K ; t]
$$

where the projective phase is a non-trivial local $\mathrm{U}(1)$ 1-cocycle. Then, to solve the Schrödinger equation

$$
i \frac{\partial}{\partial t} \Psi_{\text {phys }}[\theta, K ; t]=H \Psi_{\text {phys }}[\theta, K ; t]
$$

from (2.16) and (3.4) we obtain

$$
\tilde{\Psi}(t)=\exp \left[\frac{2 \pi i}{k} \int_{-\infty}^{t} d t^{\prime} \int d^{3} x\left((\nabla \times j)_{i} \frac{1}{\nabla^{2}} \Sigma^{0 i}\left(x, t^{\prime}\right)+(\nabla \cdot \Sigma) \frac{1}{\nabla^{2}} j^{0}\left(x, t^{\prime}\right)\right)\right]
$$

where $\Sigma_{i}=\frac{1}{2} \epsilon_{i j k} \Sigma^{j k}$.

We now substitute in the source currents (2.11) and (2.12) with the particle trajectories and string surfaces in $\mathcal{M}_{3}$ parametrized by time; i.e. $r_{a}^{0}(\tau)=\tau$ and $X_{b}^{0}\left(\sigma^{1}, \sigma^{2}\right)=\sigma^{1}$. Then using the Green's function for the 3 dimensional Laplacian on $\mathbb{R}^{3}$

$$
\left(x\left|\frac{1}{\nabla^{2}}\right| y\right)=-\frac{1}{4 \pi|x-y|}
$$


and integrating by parts over $\mathbb{R}^{3}$ the full wavefunctional is therefore

$$
\begin{aligned}
\Psi_{\text {phys }}[\theta, K ; t]=\exp & {\left[i\left\{\sum_{a} q_{a} \theta\left(r_{a}(t)\right)+\sum_{b} \phi_{b} \int d \sigma \frac{\partial X_{b}^{i}(t, \sigma)}{\partial \sigma} K_{i}\left(X_{b}(t, \sigma)\right)\right\}\right.} \\
& +\frac{i}{2 k} \sum_{a, b} q_{a} \phi_{b} \int_{-\infty}^{t} d t^{\prime} \int d \sigma \epsilon_{i j k}\left\{\dot{r}_{a}^{i}\left(t^{\prime}\right) \frac{\partial X_{b}^{k}\left(t^{\prime}, \sigma\right)}{\partial \sigma}\right. \\
& \left.\left.-\frac{\partial X_{b}^{i}\left(t^{\prime}, \sigma\right)}{\partial t^{\prime}} \frac{\partial X_{b}^{k}\left(t^{\prime}, \sigma\right)}{\partial \sigma}\right\} \frac{\left(r_{a}\left(t^{\prime}\right)-X_{b}\left(t^{\prime}, \sigma\right)\right)^{j}}{\left|r_{a}\left(t^{\prime}\right)-X_{b}\left(t^{\prime}, \sigma\right)\right|^{3}}\right]
\end{aligned}
$$

The final sum in (3.10) (i.e. $\tilde{\Psi}(t))$ is the topological linking number of the particle trajectories and the string surfaces in $\mathbb{R}^{3}$ and is the three dimensional version of the linking number in (2.9). This canonical linking is the adiabatic limit of the covariant linking found in (2.9) using the path integral approach. As a function of $t$ it is the analog of the usual 2 dimensional multivalued angle function which arises in anyon theories as the term responsible for giving charged particles anomalous exchange statistics [1]. If we look closely at the integrand of this term, we see that it has the form

$$
\frac{R_{a b} \cdot\left(d R_{a b} \times d R_{a b}\right)}{\left|R_{a b}\right|^{3}}=d \Phi_{a b}(t)
$$

where $R_{a b}=X_{b}-r_{a}$ and $\Phi_{a b}(t)$ is the solid angle formed by the string (along $X_{b}(t, \sigma)$ ) as viewed from the charged particle $\left(\right.$ at $\left.r_{a}(t)\right)$. Here the "statistics parameter" is $\frac{k}{2 \pi}$, i.e. when a particle of charge $q_{a}$ encircles a string of flux $\phi_{b}$ once, $\Psi_{\text {phys }}$ changes by the phase $\mathrm{e}^{-\frac{2 \pi i}{k} q_{a} \phi_{b}}$. The Hilbert space of BF-theory on Euclidean 3-space is therefore 1 dimensional and the wavefunctionals carry a 1 dimensional unitary representation of the fractional particle-string linking for generic values of the BF coefficient $k$.

\section{PARTICLE-STRING HOLONOMY IN CURVED SPACES}

We now move on to the more interesting case of a curved space with possibly nontrivial topology. We assume that $\mathcal{M}_{3}$ is a compact, path-connected, orientable 3-manifold without boundary. The non-trivial homology of $\mathcal{M}_{3}$ is represented by the homology groups $H_{1}\left(\mathcal{M}_{3} ; \mathbb{Z}\right)$ and $H_{2}\left(\mathcal{M}_{3} ; \mathbb{Z}\right)$, which by Poincaré-Hodge duality both have the same dimension that we denote by $p$. We shall ignore the torsion parts of the homology groups which play no role in the following. Let $\left\{L^{m}\right\}_{m=1}^{p}$ be a set of generators of $H_{1}\left(\mathcal{M}_{3} ; \mathbb{Z}\right)$ and 
$\left\{\Sigma^{m}\right\}_{m=1}^{p}$ a set of generators of $H_{2}\left(\mathcal{M}_{3} ; \mathbb{Z}\right)$. A fundamental characteristic of $\mathcal{M}_{3}$ is its linking matrix [17]

$$
M^{m n}=I_{\mathcal{M}_{3}}\left(\Sigma^{m}, L^{n}\right)=\sum_{I_{m n}} \operatorname{sgn}\left(I_{m n}\right)
$$

which counts the signed intersections $I_{m n}$ of the two dimensional surface $\Sigma^{m}$ with the one dimensional line $L^{n}$ in $\mathcal{M}_{3}$. By definition it is a topological invariant and an integer-valued matrix. Note that it is not necessarily symmetric and so it is important to keep track of the order of its two indices in what follows. Some examples of constant curvature spaces are the 3 -sphere $S^{3}$ for which $p=0$, the 3 -torus $\left(S^{1}\right)^{3}$ where $p=3$, and the product 3-manifold $S^{2} \times S^{1}$ wherein $p=1$. Notice that the linking matrix $M^{n m}$ for the latter 2 examples is just the $p \times p$ identity matrix, as is always the case for a space $\mathcal{M}_{3}$ which is the product of a one- and a two-dimensional space.

To describe the non-trivial cohomology of $\mathcal{M}_{3}$, we let $\left\{\alpha_{\ell}\right\}_{\ell=1}^{p} \in H^{1}\left(\mathcal{M}_{3} ; \mathbb{R}\right)$ be a basis of harmonic 1-forms (i.e. $\left.d \alpha_{\ell}=d * \alpha_{\ell}=0\right)$ and $\left\{\beta_{\ell}\right\}_{\ell=1}^{p} \in H^{2}\left(\mathcal{M}_{3} ; \mathbb{R}\right)$ a basis of harmonic 2 -forms which are Poincaré-duals of the homology generators

$$
\int_{L^{m}} \alpha_{\ell}=\delta_{\ell}^{m} \quad, \quad \int_{\Sigma^{m}} \beta_{\ell}=\delta_{\ell}^{m}
$$

As will become clear later on (see the Appendix, equation (A.8)), for our purposes it is necessary to choose the normalization (4.2) instead of the standard normalization usually employed wherein the harmonic generators $\alpha_{\ell}$ and $\beta_{\ell}$ are taken to be Hodge-dual basis elements [2]. Here the exterior product of these cohomology generators satisfy the reciprocal relation of the homology generators

$$
\int_{\mathcal{M}_{3}} \alpha_{\ell} \wedge \beta_{k}=M_{\ell k}
$$

where we define the inverse and determinant of the linking matrix as

$$
M_{\ell k} M^{k m}=\delta_{\ell}^{m} \quad, \quad M=\operatorname{det}\left[M^{m n}\right]
$$

In general $M_{m n}$ is rational-valued, but $M \cdot M_{m n}$ is always an integer-valued matrix. We will assume that $M$ is positive, since this can always be achieved by changing the orientation of $\mathcal{M}_{3}$ if necessary. Finally, the non-Euclidean geometry of $\mathcal{M}_{3}$ is encoded within the Hodge duality operator $*$.

The Hodge decompositions of the forms $A$ and $B$ on $\mathcal{M}_{3}$ are now as follows: The 1-form $A=A_{i} d x^{i}$ restricted to $\mathcal{M}_{3}$ can be decomposed into exact, co-exact and harmonic forms as

$$
A=d \theta+* d K^{\prime}+a^{\ell} \alpha_{\ell}
$$


where the scalar field $\theta$ and 1 -form field $K^{\prime}$ satisfy

$$
\nabla_{g}^{2} \theta=* d * A, \quad \Phi^{\prime}=* d * d K^{\prime}=* d A
$$

and the harmonic coefficients are given by

$$
a^{\ell}(t)=M^{m \ell} \int_{\mathcal{M}_{3}} A \wedge \beta_{m}=\int_{L^{\ell}} A
$$

Here $\nabla_{g}$ denotes the covariant derivative with respect to the metric $g$ of $\mathcal{M}_{3}$ and $\nabla_{g}^{2}$ is the corresponding scalar Laplacian. Likewise the 2 -form $B=\frac{1}{2} B_{i j} d x^{i} \wedge d x^{j}$ can be decomposed on $\mathcal{M}_{3}$ as

$$
B=d K+* d \theta^{\prime}+b^{\ell} \beta_{\ell}
$$

where the scalar field $\theta^{\prime}$ and 1 -form field $K$ obey

$$
\nabla_{g}^{2} \theta^{\prime}=* d B, \quad d * d K=d * B
$$

and the harmonic coefficients are

$$
b^{\ell}(t)=M^{\ell m} \int_{\mathcal{M}_{3}} B \wedge \alpha_{m}=\int_{\Sigma^{\ell}} B
$$

Since by assumption $\int_{\mathcal{M}_{3}} d^{3} x \nabla_{g} \cdot B=0$, the function $* d B$ in (4.9) contains no zero modes, or the constant function in this case, of the scalar Laplacian $\nabla_{g}^{2}$. Similarily the 1 -form $* d A$ in (4.6) contains no zero modes of the Laplacian $\left(\nabla_{g}^{(1)}\right)^{2}$ acting on 1-forms. Moreover, we can set the zero modes of $d * B=\nabla_{g} \times B$ and $* d * A=\nabla_{g} \cdot A$ to zero using time-independent gauge transformations. The harmonic parts (4.7) and (4.10) of the fields represent the additional degrees of freedom of $A$ and $B$ that are present when $\mathcal{M}_{3}$ is homologically non-trivial (compare with equations (3.1) and (3.2) of Section 3).

The gauge constraints (2.13) are again solved by the decomposition (3.5) of the wavefunctions. Substituting (4.5)-(4.10) into the remaining action, without the gauge constraints, in (2.14) and integrating by parts over $\mathcal{M}_{3}$ gives

$$
S=\int d t\left[\int_{\mathcal{M}_{3}}\left(\frac{k}{2 \pi}\left(-\dot{\theta} \nabla_{g}^{2} \theta^{\prime}+\dot{\Phi}_{i}^{\prime} K^{i}\right) d^{3} x+A \wedge * \tilde{j}+B \wedge * \tilde{\Sigma}\right)+\frac{k}{2 \pi} \dot{a}^{\ell} M_{\ell m} b^{m}\right]
$$

where $\tilde{j}$ and $\tilde{\Sigma}$ are the dual forms, over $\mathcal{M}_{3}$, of the particle and string current vector fields (2.11) and (2.12), respectively. From (4.11) we read off the non-vanishing canonical commutators

$$
\left[\theta(x), \nabla_{g}^{2} \theta^{\prime}(y)\right]=-\frac{2 \pi i}{k} \delta^{(3)}(x, y) \quad, \quad\left[K_{i}(x), \Phi_{j}^{\prime}(y)\right]=-\frac{2 \pi i}{k} P_{i j} \delta^{(3)}(x, y)
$$




$$
\left[a^{\ell}, b^{m}\right]=\frac{2 \pi i}{k} M^{\ell m}
$$

where $P_{i j}$ is the transverse projection operator satisfying

$$
\nabla_{g}^{i} P_{i j}=\nabla_{g}^{j} P_{i j}=0 \quad, \quad P_{i j} A^{j}=A_{i}-\left(\nabla_{g}\right)_{i}\left(\frac{1}{\left(\nabla_{g}^{2}\right)^{\prime}} \nabla_{g}^{j} A_{j}\right)
$$

and the prime on the Laplacian indicates that we remove its zero modes.

In the Schrödinger picture we therefore consider the wavefunctions $\Psi_{\text {phys }}[\theta, K, a ; t]$ and we can represent the commutation relations (4.12) and (4.13) by the derivative operators

$$
\begin{gathered}
\theta^{\prime}(x)=\frac{2 \pi i}{k} \frac{1}{\left(\nabla_{g}^{2}\right)^{\prime}} \frac{\delta}{\delta \theta(x)} \quad, \quad \Phi_{i}^{\prime}(x)=\frac{2 \pi i}{k} P_{i j} \frac{\delta}{\delta K_{j}(x)} \\
b^{m}=-\frac{2 \pi i}{k} M^{\ell m} \frac{\partial}{\partial a^{\ell}}
\end{gathered}
$$

As we shall see, the projection operator $P_{i j}$ ensures the invariance of the wavefunctions under the time-independent longitudinal symmetry $K \rightarrow K+d \Lambda$, where $\Lambda$ is an arbitrary function, which is dual to the string current symmetry $\tilde{\Sigma} \rightarrow \tilde{\Sigma}+* d \tilde{\Lambda}$, where $\tilde{\Lambda}$ is an arbitrary 1-form. This symmetry arises from the continuity equation for $\Sigma^{\mu \nu}$.

\subsection{Source-free Case: Effects of Non-trivial \\ Topology and Cohomology Representations}

When $\mathcal{M}_{3}$ has non-trivial homology, the coefficients (4.7) and (4.10) of the harmonic parts of the gauge fields transform under the large gauge transformations which arise from windings around the non-trivial homology cycles of $\mathcal{M}_{3}$ (i.e. $n, m \neq 0$ in (2.5)). The physical state wavefunctions should respect these global gauge symmetries just as they respect the local gauge symmetries generated by (2.13) (see (3.6)), and this constraint then partitions the physical Hilbert space into superselection sectors labelled by the homology classes of $\mathcal{M}_{3}$. To see how this works, we first consider this model without sources. Then the constraints (2.13) imply that $A$ and $B$ restricted to $\mathcal{M}_{3}$ are closed forms. Consequently, the harmonic coefficients (4.7) and (4.10) are topological invariants which generate the signed intersection number (4.1) through the operator algebra $(4.13)^{3}$.

Had we instead chosen the normalization of the harmonic generators corresponding to Hodge duality, i.e. $\int_{\mathcal{M}_{3}} \alpha_{\ell} \wedge \beta_{k}=\delta_{\ell k}$, then the operator algebra of $a^{\ell}$ and $b^{m}$ would involve the identity matrix $\delta^{\ell m}$ rather than the linking matrix $M^{\ell m}$. However, because of the canonical commutator (2.15), the contour and surface integrals of $A$ and $B$, respectively, would still satisfy the algebra (4.13). 
With no sources present the Hamiltonian (2.16) vanishes and so the wavefunctions are time-independent. Moreover, the local gauge constraints (2.13) and the relations (4.5), (4.8), and (4.15) imply that

$$
\frac{\delta}{\delta \theta} \Psi_{\text {phys }}[\theta, K, a]=P_{i j} \frac{\delta}{\delta K_{j}} \Psi_{\text {phys }}[\theta, K, a]=0
$$

so that the physical states depend only on the global harmonic coefficients $a^{\ell}$. It remains to solve for the invariance under the time-independent large gauge transformations (2.2) and (2.3) satisfying (2.5), which in terms of the harmonic coefficients (4.7) and (4.10) are given by the translations

$$
a^{\ell} \rightarrow a^{\ell}+2 \pi n^{\ell} \quad, \quad b^{\ell} \rightarrow b^{\ell}+2 \pi m^{\ell} \quad ; \quad n^{\ell}, m^{\ell} \in \mathbb{Z}
$$

It is convenient here to instead work in a holomorphic polarization defined by the complex variables ${ }^{4}$

$$
\gamma^{\ell}=a^{\ell}+M^{m \ell} \rho_{m k} b^{k} \quad, \quad \bar{\gamma}^{\ell}=a^{\ell}+M^{m \ell} \bar{\rho}_{m k} b^{k}
$$

where $\rho=\left[\rho_{m k}\right]$ is an arbitrary symmetric $p \times p$ complex-valued matrix whose imaginary part is negative-definite. The gauge transformations (4.18) in these new variables are

$$
\gamma^{\ell} \rightarrow \gamma^{\ell}+2 \pi\left(n^{\ell}+M^{m \ell} \rho_{m k} m^{k}\right) \quad, \quad \bar{\gamma}^{\ell} \rightarrow \bar{\gamma}^{\ell}+2 \pi\left(n^{\ell}+M^{m \ell} \bar{\rho}_{m k} m^{k}\right)
$$

and the commutation relations (4.13) become

$$
\left[\gamma^{\ell}, \gamma^{k}\right]=\left[\bar{\gamma}^{\ell}, \bar{\gamma}^{k}\right]=0 \quad, \quad\left[\gamma^{\ell}, \bar{\gamma}^{k}\right]=-\frac{2 \pi}{k} \Omega^{\ell k}
$$

where

$$
\Omega^{\ell k}=-2 M^{p \ell} \operatorname{Im} \rho_{p q} M^{q k}
$$

is a real-valued positive-definite symmetric matrix. These relations may be represented by

$$
\bar{\gamma}^{\ell}=\frac{2 \pi}{k} \Omega^{\ell k} \frac{\partial}{\partial \gamma^{k}}
$$

and in the coordinates (4.19) we will instead be looking for the coherent state wavefunctions which we denote by $\Psi_{0}(\gamma)$.

4 This polarization eludes the delta-function singularities which appear in the wavefunctions when the usual Schrödinger polarization is used. 
The matrix $\rho$ introduced above can be interpreted as follows. The phase space of the source-free BF theory is the $2 p$ dimensional space of the $a^{\ell}$ and $b^{\ell}$ variables

$$
\mathcal{P}=H^{1}\left(\mathcal{M}_{3} ; \mathbb{R}\right) \oplus H^{2}\left(\mathcal{M}_{3} ; \mathbb{R}\right)
$$

$\rho$ can then be thought of as parametrizing a complex structure on $\mathcal{P}$ forming the $p$ dimensional complex space of the $\gamma^{\ell}$ variables, and this then determines all of the topological degrees of freedom which remain in the source-free case modulo the large gauge transformations (4.20). In fact, as we shall see later on, the positive-definite symmetric matrix $\Omega$ actually defines a metric on $\mathcal{P}$, and from its definition (4.22) (and the definition (4.19)) we see that it incorporates the topological linking of the homology cycles of $\mathcal{M}_{3}$. However, since the action (2.1) defines a topological field theory, all observables will be independent of this phase space complex structure. This is analogous to the situation in Chern-Simons theory [21]. From (4.24) and (4.19) we see that the quantization of the classical phase space of the BF system will give (projective) quantum representations of the cohomology groups of $\mathcal{M}_{3}$.

From (4.21) it follows that the quantum operators which generate the global gauge transformations (4.20) in the Schrödinger picture are

$$
U(n, m)=\exp \left[2 \pi\left(n^{\ell}+M^{m \ell} \rho_{m k} m^{k}\right) \frac{\partial}{\partial \gamma^{\ell}}-k\left(n^{\ell}+M^{m \ell} \bar{\rho}_{m k} m^{k}\right)\left(\Omega^{-1}\right)_{\ell q} \gamma^{q}\right]
$$

The unitary operators (4.25) in general do not commute among themselves, in contrast with their classical counterparts, and the Baker-Campbell-Hausdorff formula

$$
\mathrm{e}^{X+Y}=\mathrm{e}^{-[X, Y] / 2} \mathrm{e}^{X} \mathrm{e}^{Y}
$$

along with the commutation relations (4.21) show that the algebra of these large gauge transformations is a variation of a clock algebra

$$
U(n, m) U\left(n^{\prime}, m^{\prime}\right)=\mathrm{e}^{2 \pi i k\left(n^{\ell} M_{\ell n} m^{n}-n^{\ell} M_{\ell n} m^{\prime n}\right)} U\left(n^{\prime}, m^{\prime}\right) U(n, m)
$$

This U(1) 2-cocycle relation differs from the standard clock algebra in that the matrix $M_{\ell n}$ in (4.27) is usually the identity matrix $\delta_{\ell n}$. On 3-manifolds $\mathcal{M}_{3}$ for which the linking matrix is not trivially the identity matrix the 2-cocycle appearing in (4.27) is quite natural, since it then also reflects the possible non-trivial linkings of the homology cycles of $\mathcal{M}_{3}$ which should be represented by the generators of windings around them. To determine the effect of these generators explicitly on the wavefunctions $\Psi_{0}(\gamma)$, we separate out the derivative 
part of the operator (4.25) using the Baker-Campbell-Hausdorff formula (4.26) again, and we find that the behaviour of the wavefunctions under large gauge transformations is

$$
\begin{gathered}
U(n, m) \Psi_{0}\left(\gamma^{\ell}\right)=\exp \left[-k\left(n^{\ell}+M^{m \ell} \bar{\rho}_{m k} m^{k}\right)\left(\Omega^{-1}\right)_{\ell q} \gamma^{q}-\pi k\left(n^{\ell}+M^{m \ell} \bar{\rho}_{m k} m^{k}\right)\left(\Omega^{-1}\right)_{\ell q}\right. \\
\left.\times\left(n^{q}+M^{r q} \rho_{r s} m^{s}\right)\right] \Psi_{0}\left(\gamma^{\ell}+2 \pi\left(n^{\ell}+M^{m \ell} \rho_{m k} m^{k}\right)\right)
\end{gathered}
$$

When the coupling constant $\frac{1}{k}$ is an irrational number, the algebra (4.27) of large gauge transformations is infinite dimensional. Finite dimensional representations of the non-trivial homology of $\mathcal{M}_{3}$ can however be obtained when the BF coefficent $k$ is either a positive integer or rational number, $k=M \frac{k_{1}}{k_{2}} ; k_{1}, k_{2} \in \mathbb{Z}^{+}$(Negative $k$ can then be obtained by reversing the orientation of $\mathcal{M}_{3}$ and thus changing the sign of $M$ ). We shall assume these discrete values of $k$ for the remainder of this Paper. Then for any set of integers $(n, m)$, the algebra (4.27) implies that $U\left(k_{2} n, k_{2} m\right)$ commutes with all of the other gauge transformation generators, as then the phase in (4.27) is an integer multiple of $2 \pi$. Since these operators are unitary and commute with the (zero) Hamiltonian here, it follows that their action (4.28) on the wavefunctions must lie on the same ray in the Hilbert space as that defined by $\Psi_{0}(\gamma)$, i.e.

$$
U\left(k_{2} n, k_{2} m\right) \Psi_{0}(\gamma)=\mathrm{e}^{i \eta_{(n, m)}} \Psi_{0}(\gamma)
$$

for some phases $\eta_{(n, m)} \in S^{1}$. Comparing this with (4.28) we see that the wavefunctions enjoy the quasi-periodicity property

$$
\begin{aligned}
& \Psi_{0}\left(\gamma^{\ell}+2 \pi k_{2}\left(n^{\ell}+M^{m \ell} \rho_{m k} m^{k}\right)\right)=\exp \left[i \eta_{(n, m)}+k_{1}\left(n^{\ell}+M^{m \ell} \bar{\rho}_{m k} m^{k}\right)\left(\Omega^{-1}\right) \ell_{\ell} \gamma^{q}\right. \\
& \left.+\pi k_{1} k_{2}\left(n^{\ell}+M^{m \ell} \bar{\rho}_{m k} m^{k}\right)\left(\Omega^{-1}\right)_{\ell q}\left(n^{q}+M^{r q} \rho_{r s} m^{s}\right)\right] \Psi_{0}\left(\gamma^{\ell}\right)
\end{aligned}
$$

The only functions which obey quasi-periodic conditions such as (4.30) are combinations of the Jacobi theta functions [22]

$$
\Theta\left(\begin{array}{c}
c \\
d
\end{array}\right)(z \mid \Pi)=\sum_{\left\{n_{\ell}\right\} \in \mathbb{Z}^{p}} \exp \left[i \pi\left(n^{\ell}+c^{\ell}\right) \Pi_{\ell k}\left(n^{k}+c^{k}\right)+2 \pi i\left(n^{\ell}+c^{\ell}\right)\left(z_{\ell}+d_{\ell}\right)\right]
$$

where $c^{\ell}, d_{\ell} \in[0,1]$ and $z_{\ell} \in \mathbb{C}$. The functions (4.31) are well-defined holomorphic functions of $\left\{z_{\ell}\right\} \in \mathbb{C}^{p}$ for $\Pi=\left[\Pi_{\ell k}\right]$ in the Siegal upper half-plane, and they obey the doubly semi-periodic conditions

$$
\Theta\left(\begin{array}{c}
c \\
d
\end{array}\right)\left(z_{\ell}+s_{\ell}+\Pi_{\ell k} t^{k} \mid \Pi\right)=\exp \left[2 \pi i c^{\ell} s_{\ell}-i \pi t^{\ell} \Pi_{\ell k} t^{k}-2 \pi i t^{\ell}\left(z_{\ell}+d_{\ell}\right)\right] \Theta\left(\begin{array}{c}
c \\
d
\end{array}\right)(z \mid \Pi)
$$


where $s_{\ell}$ and $t^{\ell}$ are integers, and

$$
\Theta\left(\begin{array}{c}
c \\
d
\end{array}\right)\left(z_{\ell}+C \Pi_{\ell k} t^{k} \mid \Pi\right)=\exp \left[-i \pi C^{2} t^{\ell} \Pi_{\ell k} t^{k}-2 \pi i C t^{\ell}\left(z_{\ell}+d_{\ell}\right)\right] \Theta\left(\begin{array}{c}
c+C t \\
d
\end{array}\right)(z \mid \Pi)
$$

for any non-integer $C \in \mathbb{R}$. It should be emphasized that the transformations (4.32) can be performed in many different steps with the same final result, but successive applications of (4.32) and (4.33) do not commute. Instead, when applied in different orders, the final results differ by a phase which forms a representation of the algebra (4.27). We define the operators $U(n, m)$ with the convention that the transformation (4.32) is applied before (4.33).

After some algebra, we find that the algebraic constraints (4.30) are uniquely solved by the wavefunctions

$$
\Psi_{0}^{(q)}\left(\begin{array}{c}
c \\
d
\end{array}\right)(\gamma)=\mathrm{e}^{\frac{k}{4 \pi} \gamma^{\ell}\left(\Omega^{-1}\right)_{\ell k} \gamma^{k}} \Theta\left(\begin{array}{c}
\frac{c+q}{M k_{1} k_{2}} \\
d
\end{array}\right)\left(\frac{M k_{1}}{2 \pi} M_{m \ell} \gamma^{m} \mid-k_{1} k_{2} M \rho\right)
$$

where $q^{\ell}=1,2, \ldots, k_{1} k_{2} M$. Computing the periodic behaviour of (4.34) we find that the phases in (4.29) are given by the non-trivial 1-cocycle

$$
\frac{\eta_{(n, m)}}{2 \pi}=\alpha_{1}(n, m)=c^{\ell} M_{m \ell} n^{m}+d_{\ell} m^{\ell}-\frac{1}{2} k_{1} k_{2} M n^{m} M_{m \ell} m^{\ell}
$$

Under an arbitrary large gauge transformation (4.28) we have

$$
\begin{aligned}
U(n, m) \Psi_{0}^{(q)}\left(\begin{array}{l}
c \\
d
\end{array}\right)(\gamma) & =\exp \left[\frac{2 \pi i}{k_{2}}\left(c^{\ell} M_{m \ell} n^{m}+d_{\ell} m^{\ell}\right)-i \pi k n^{m} M_{m \ell} m^{\ell}+\frac{2 \pi i}{k_{2}} q^{\ell} M_{m \ell} n^{m}\right] \\
\times \Psi_{0}^{\left(q^{\ell}-k_{1} M m^{\ell}\right)}\left(\begin{array}{c}
c \\
d
\end{array}\right)(\gamma) & \\
= & \sum_{q^{\prime}}[U(n, m)]_{q q^{\prime}} \Psi_{0}^{\left(q^{\prime}\right)}\left(\begin{array}{c}
c \\
d
\end{array}\right)(\gamma)
\end{aligned}
$$

where the unitary matrices

$[U(n, m)]_{q q^{\prime}}=\exp \left[\frac{2 \pi i}{k_{2}}\left(c^{\ell} M_{m \ell} n^{m}+d_{\ell} m^{\ell}\right)-i \pi k n^{m} M_{m \ell} m^{\ell}+\frac{2 \pi i}{k_{2}} q^{\ell} M_{m \ell} n^{m}\right] \delta_{q^{\ell}-k_{1} M m^{\ell}, q^{\prime k}}$

form a $\left(k_{2}\right)^{p}$ dimensional projective representation, which is cyclic of period $k_{2}$, of the algebra (4.27) of large gauge transformations. The projective phase here is the non-trivial global U(1) 1-cocycle

$$
\alpha_{1}^{(q)}(n, m)=\frac{1}{k_{2}}\left(c^{\ell} M_{r \ell} n^{r}+d_{\ell} m^{\ell}+q^{\ell} M_{r \ell} n^{r}-\frac{M k_{1}}{2} n^{r} M_{r \ell} m^{\ell}\right)
$$


Therefore, the Hilbert space of source-free BF-theory, on spaces with $p$ dimensional first and second homology and linking matrix $M^{k \ell}$, is $\left(k_{1} k_{2} M\right)^{p}$ dimensional and the wavefunctions carry a $\left(k_{2}\right)^{p}$ dimensional representation of the corresponding discrete group of large gauge transformations. Notice that the possible non-trivial homology linking is even represented directly in the dimensionality of the Hilbert space.

\subsection{Inclusion of Sources: Fractional Statistics and Duality}

In the last Subsection we have seen that on homologically non-trivial spaces, the Hilbert space of physical states is non-trivial even in the absence of particle and string sources (in contrast to the simply connected case), due to the existence of non-trivial global gauge transformations. To obtain representations of the particle-string holonomy in $\mathcal{M}_{3}$, we reintroduce sources into the problem and Hodge decompose the dual current forms $\tilde{j}=j_{i} d x^{i}$ and $\tilde{\Sigma}=\frac{1}{2} \Sigma_{i j} d x^{i} \wedge d x^{j}$ as before on $\mathcal{M}_{3}$. With the same conventions for the particle trajectories and string surfaces as in Section 3 above, we have

$$
\tilde{j}=d \omega^{\prime}+* d \Omega+j_{\ell} M^{m \ell} * \beta_{m}
$$

where from the continuity equation $\partial_{\mu} j^{\mu}=0$

$$
* d * \tilde{j}=\nabla_{g}^{2} \omega^{\prime}=-\partial_{t} j^{0} \quad, \quad d * d \Omega=d \tilde{j}
$$

and from the explicit form (2.11) of the particle current

$$
j_{\ell}(t)=\sum_{a} q_{a} \dot{r}_{a}^{i}(t)\left(\alpha_{\ell}\right)_{i}\left(r_{a}(t)\right)
$$

Similarily

$$
\tilde{\Sigma}=d \Pi^{\prime}+* d \pi+\Sigma_{\ell} M^{\ell m} * \alpha_{m}
$$

where from the conservation law $\partial_{\mu} \Sigma^{\mu \nu}=0$

$$
* d * \tilde{\Sigma}=* d * d \Pi^{\prime}=\partial_{t} \Sigma_{0}, \quad \nabla_{g}^{2} \pi=* d \tilde{\Sigma}
$$

where $\Sigma_{0}=\Sigma_{0 i} d x^{i}$, and the explicit expression (2.12) for the string current implies

$$
\Sigma_{\ell}(t)=\sum_{b} \phi_{b} \int d \sigma \frac{\partial X_{b}^{i}(t, \sigma)}{\partial t} \frac{\partial X_{b}^{j}(t, \sigma)}{\partial \sigma}\left(\beta_{\ell}\right)_{i j}\left(X_{b}(t, \sigma)\right)
$$


As for the gauge fields, we can eliminate the zero modes of the appropriate Laplacian operators using our previous assumptions and the symmetries generated by the continuity equations for the sources.

Substituting all of the above Hodge decompositions into (2.16) and integrating by parts over $\mathcal{M}_{3}$, the Hamiltonian can be written as

$$
H=\int_{\mathcal{M}_{3}} d^{3} x\left(-\theta \frac{\partial j^{0}}{\partial t}-K_{i} \frac{\partial \Sigma^{0 i}}{\partial t}-\Omega_{i} \Phi^{i}+\pi(x) \nabla_{g}^{2} \theta^{\prime}\right)-\left(a^{\ell} j_{\ell}+b^{\ell} \Sigma_{\ell}\right)
$$

and in the Schrödinger picture we can use the representations (4.15) and (4.16) to write

$$
H=\int_{\mathcal{M}_{3}} d^{3} x\left[\left(-\theta \frac{\partial j^{0}}{\partial t}+\frac{2 \pi i}{k} \pi(x) \frac{\delta}{\delta \theta}\right)+\left(-K_{i} \frac{\partial \Sigma^{0 i}}{\partial t}-\frac{2 \pi i}{k} \Omega_{i} P_{j}^{i} \frac{\delta}{\delta K_{j}}\right)\right]+H_{T}
$$

where the topological Hamiltonian $H_{T}$, written in terms of the holomorphic polarization (4.19) and the representation (4.23), is

$$
H_{T}=i\left(\Sigma_{n}-\bar{\rho}_{n m} M^{m \ell} j_{\ell}\right) M^{n p}\left(\Omega^{-1}\right)_{p k} \gamma^{k}-\frac{2 \pi i}{k}\left(\Sigma_{n}-\rho_{n m} M^{m \ell} j_{\ell}\right) M^{n k} \frac{\partial}{\partial \gamma^{k}}
$$

We see that the Hamiltonian separates into three commuting pieces, one depending only on the local exact part of $A$, one on the local exact part of $B$, and the other depending only on the global harmonic parts of the fields. The Schrödinger equation (3.7) can therefore be solved by separating the variables $\theta, K$ and $\gamma$ to get the wavefunctions $\Psi_{\text {phys }}[\theta, K, \gamma ; t]=$ $\Psi_{L}[\theta, K ; t] \Psi_{T}(\gamma ; t)$.

The local wavefunctionals $\Psi_{L}$ must solve the local gauge constraints $(2.13)$, which as in (3.5) are solved in the form

$$
\Psi_{L}[\theta, K ; t]=\exp \left[i \int_{\mathcal{M}_{3}} d^{3} x\left(\theta(x) j^{0}(x, t)+K_{i}(x) \Sigma^{0 i}(x, t)\right)\right] \tilde{\Psi}_{L}(t)
$$

and they represent the local gauge symmetries through a 1 dimensional projective representation as discussed in Section 3. The remaining piece $\tilde{\Psi}_{L}$ is found by substituting (4.48) and (4.46) into the Schrödinger equation (3.7), from which we find

$$
\tilde{\Psi}_{L}(t)=\exp \left[\frac{2 \pi i}{k} \int_{-\infty}^{t} d t^{\prime} \int_{\mathcal{M}_{3}} d^{3} x\left(\pi\left(x, t^{\prime}\right) j^{0}\left(x, t^{\prime}\right)-\Omega_{i}\left(x, t^{\prime}\right) \Sigma^{0 i}\left(x, t^{\prime}\right)\right)\right]
$$

The evaluation of this integral is done in the Appendix. It is found that

$$
\tilde{\Psi}_{L}(t)=\exp \left[-\frac{i}{2 k} \sum_{a, b} q_{a} \phi_{b} \int_{-\infty}^{t} d t^{\prime} \frac{d \Phi_{a b}^{(g)}\left(t^{\prime}\right)}{d t^{\prime}}+\frac{2 \pi i}{k} \int_{-\infty}^{t} j_{\ell}\left(t^{\prime}\right) d t^{\prime} M^{m \ell} \int_{-\infty}^{t^{\prime}} \Sigma_{m}\left(t^{\prime \prime}\right) d t^{\prime \prime}\right]
$$


where $\Phi_{a b}^{(g)}(t)=\Phi^{(g)}\left(r_{a}^{i}(t), X_{b}^{j}(t, \sigma)\right)$ is the generalization of the solid angle (3.11) to a curved space (see the Appendix, equation (A.20)). Everytime a particle goes around a loop, or a loop goes around a particle in the opposite direction, $\Phi^{(g)}$ increases by $4 \pi$. Thus we recover the fractional linking of particles and strings found earlier in Euclidean 3-space. The canonical linking function $\Phi^{(g)}(t)$ is the adiabatic limit of the standard covariant particle-string linking in a general 4-manifold $\mathcal{M}_{4}[2,3,5]$.

The solution $\Psi_{T}$ of the Schrödinger equation determined by the topological Hamiltonian $H_{T}$ in (4.47) is immediate

$$
\begin{aligned}
\Psi_{T}(\gamma ; t)=\exp \left[\int_{-\infty}^{t}\right. & \left(\Sigma_{n}\left(t^{\prime}\right)-\bar{\rho}_{n m} M^{m \ell} j_{\ell}\left(t^{\prime}\right)\right) d t^{\prime} M^{n p}\left(\Omega^{-1}\right)_{p k} \gamma^{k} \\
& -\frac{2 \pi}{k} \int_{-\infty}^{t}\left(\Sigma_{n}\left(t^{\prime}\right)-\rho_{n m} M^{m \ell} j_{\ell}\left(t^{\prime}\right)\right) d t^{\prime} M^{n p} \\
& \left.\times\left(\Omega^{-1}\right)_{p q} M^{k q} \int_{-\infty}^{t^{\prime}}\left(\Sigma_{k}\left(t^{\prime \prime}\right)-\bar{\rho}_{k r} M^{r s} j_{s}\left(t^{\prime \prime}\right)\right) d t^{\prime \prime}\right] \\
& \times \Psi_{0}^{(q)}\left(\begin{array}{c}
c \\
d
\end{array}\right)\left(\gamma^{k}-\frac{2 \pi}{k} M^{n k} \int_{-\infty}^{t}\left(\Sigma_{n}\left(t^{\prime}\right)-\rho_{n m} M^{m \ell} j_{\ell}\left(t^{\prime}\right)\right) d t^{\prime}\right)
\end{aligned}
$$

The last function $\Psi_{0}$ in (4.51) is at first glance arbitrary since the combination $\gamma^{k}-$ $\frac{2 \pi}{k} M^{n k} \int_{-\infty}^{t}\left(\Sigma_{n}\left(t^{\prime}\right)-\rho_{n m} M^{m \ell} j_{\ell}\left(t^{\prime}\right)\right) d t^{\prime}$ in its argument leads directly to a solution of the equation

$$
\frac{\partial \Psi_{0}}{\partial t}=-\frac{2 \pi}{k}\left(\Sigma_{n}-\rho_{n m} M^{m \ell} j_{\ell}\right) M^{n k} \frac{\partial \Psi_{0}}{\partial \gamma^{k}}
$$

which arises from the Schrödinger equation after factoring out the exponential term in (4.51). However, when there are no particles and strings present we find that $\Psi_{T}(\gamma)$ must reduce to the coherent state wavefunctions $\Psi_{0}(\gamma)$ discussed in Subsection 4.1 above, which we know to be the functions $\Psi_{0}^{(q)}$ given by (4.34). (4.51) determines that part of the full wavefunction which represents the source currents traversing along the nontrivial homology cycles in $\mathcal{M}_{3}$, as well as the global gauge symmetries through the multidimensional projective representation discussed in Subsection 4.1.

When we put together $\Psi_{L}$ and $\Psi_{T}$ above, use the equation (4.34) for $\Psi_{0}^{(q)}$, and substitute in the expressions (2.11) and (2.12) for the source currents, we obtain the full 
wavefunction

$$
\begin{aligned}
\Psi_{\text {phys }}^{(q)}\left(\begin{array}{c}
c \\
d
\end{array}\right)[\theta, & K, \gamma ; t]=\exp \left[i \sum_{a} q_{a} \theta\left(r_{a}(t)\right)+i \sum_{b} \phi_{b} \int d \sigma \frac{\partial X_{b}^{i}(t, \sigma)}{\partial \sigma} K_{i}\left(X_{b}(t, \sigma)\right)\right] \\
& \times \exp \left[-\frac{i}{2 k} \sum_{a, b} q_{a} \phi_{b}\left(\Phi_{a b}^{(g)}(t)-\Phi_{a b}^{(g)}(-\infty)\right)+\frac{k}{4 \pi} \gamma^{\ell}\left(\Omega^{-1}\right) \ell k \gamma^{k}\right. \\
& -i \gamma^{k} \int_{-\infty}^{t} j_{k}\left(t^{\prime}\right) d t^{\prime}+\frac{2 \pi i}{k} \int_{-\infty}^{t} j_{\ell}\left(t^{\prime}\right) d t^{\prime} M^{m \ell} \int_{-\infty}^{t} \Sigma_{m}\left(t^{\prime}\right) d t^{\prime} \\
& \left.-\frac{i \pi}{k} \int_{-\infty}^{t} j_{k}\left(t^{\prime}\right) d t^{\prime} M^{p k} \rho_{p q} M^{q \ell} \int_{-\infty}^{t} j_{\ell}\left(t^{\prime}\right) d t^{\prime}\right] \Theta\left(\frac{c+q}{k_{1} k_{2} M}\right)\left(\frac{M k_{1}}{2 \pi} M_{k n} \gamma^{k}\right. \\
& \left.-k_{2} \int_{-\infty}^{t}\left(\Sigma_{n}\left(t^{\prime}\right)-\rho_{n m} M^{m \ell} j_{\ell}\left(t^{\prime}\right)\right) d t^{\prime} \mid-k_{1} k_{2} M \rho\right)
\end{aligned}
$$

As shown in the Appendix, the wavefunctions (4.53) are independent of the particular paths of motion of the particles and strings, as long as these do not intersect. They do, however, depend on their configurations through the local parts of the gauge fields, the topological current integrals and the angle function $\Phi_{a b}^{(g)}(t)-\Phi_{a b}^{(g)}(-\infty)$.

Notice that the states (4.53) also contain $2 p$ free parameters $c$ and $d$. However, it can be observed that

$$
\Psi_{0}^{(q)}\left(\begin{array}{c}
c \\
d
\end{array}\right)(-\gamma)=(-1)^{4 c^{\ell} d_{\ell}} \Psi_{0}^{(q)}\left(\begin{array}{c}
c \\
d
\end{array}\right)(\gamma)
$$

only when $c^{\ell}, d_{\ell} \in\left\{0, \frac{1}{2}\right\}$. For other parameter values the reflection symmetry $\gamma \rightarrow-\gamma$ does not close in the set of functions (4.34). The values $c^{\ell}, d_{\ell} \in\left\{0, \frac{1}{2}\right\}$ correspond to a choice of spin structure on the complex multi-torus formed by the topological phase space $\mathcal{P}$ modulo large gauge transformations. This spin structure increases the number of wavefunctions (4.53) by $4^{p}$. We have not found any other symmetry of the theory, for example a symmetry that relates different complex structures on $\mathcal{P}$, which would select special values for $c$ and $d$, and it would be interesting to investigate this further.

The final step in constructing the Hilbert space of the particle-string system is to determine the transition amplitudes between physical states. The inner product in the finite-dimensional vector space spanned by the wavefunctions (4.53) is given by

$$
\left(\Psi_{1}, \Psi_{2}\right)=\int D \theta D K<\Psi_{1}[\theta, K], \Psi_{2}[\theta, K]>_{\mathcal{P}}
$$

where the inner product on the topological phase space (4.24) is defined by the usual 
coherent state measure for the holomorphic polarization (4.19) [23]

$$
<\Psi_{1}, \Psi_{2}>_{\mathcal{P}}=\int_{P} \prod_{\ell=1}^{p} d \gamma^{\ell} d \bar{\gamma}^{\ell} \operatorname{det} \Omega^{-1} \mathrm{e}^{-\frac{k}{2 \pi} \gamma^{k}\left(\Omega^{-1}\right)_{k \ell} \bar{\gamma}^{\ell}} \Psi_{1}^{*}(\bar{\gamma}) \Psi_{2}(\gamma)
$$

In the subspace spanned by the wavefunctions (4.34), wherein the integrand of (4.56) is completely invariant under the large gauge transformations (4.20), the integration in (4.56) can be restricted to the plaquette $P=\left\{\gamma^{\ell}=u^{\ell}+M^{m \ell} \rho_{m k} v^{k}: u^{\ell}, v^{\ell} \in[0,1]\right\}$, the multitorus which is the reduced phase space of the $\gamma$ 's, after dividing out by the volume of the gauge group in (4.55). With the measure (4.56) and the commutation relations (4.21), we find that $\gamma^{\dagger}=\bar{\gamma}$, as it should be, and also that the infinitesimal variation norm is given by $\|\delta \gamma\|^{2}=(\delta \gamma)^{k}\left(\Omega^{-1}\right)_{k \ell}(\delta \bar{\gamma})^{\ell}$. Thus the matrix $\Omega$ given by (4.22) defines a metric on the topological phase space $\mathcal{P}$ of harmonic forms. Notice that the wavefunctions (4.34) have inner product

$$
<\Psi_{0}^{(q)}, \Psi_{0}^{\left(q^{\prime}\right)}>_{\mathcal{P}}=\operatorname{det}^{-1 / 2} \Omega \delta^{q q^{\prime}}
$$

and so the basis (4.53) can be used to define an orthonormal basis of the full physical Hilbert space.

When the current sources are transported around contractable cycles in $\mathcal{M}_{3}$, there is no additional phase contribution to the wavefunctions (4.53). This is not true, however, for homologically non-trivial motions of the particles and strings. Let us consider the effect on the wavefunctions (4.53) of the motion of particles and strings whereby first the particles wind $t_{k}$ times around the $k$-th homology 1-cycle, so that $\int_{-\infty}^{\tilde{t}} j_{k}\left(t^{\prime}\right) d t^{\prime}=t_{k}$ and $\int_{-\infty}^{\tilde{t}} \Sigma^{k}\left(t^{\prime}\right) d t^{\prime}=0$, and then afterwards the strings wind $s_{k}$ times around the $k$-th homology 2-cycle, so that $\int_{\tilde{t}}^{t} j_{k}\left(t^{\prime}\right) d t^{\prime}=0$ and $\int_{\tilde{t}}^{t} \Sigma^{k}\left(t^{\prime}\right) d t^{\prime}=s_{k}$. The linkings of these particles and strings are represented by the angle function $\Phi_{a b}^{(g)}$. Then we find, modulo these linkings, from (4.33) that the wavefunctions (4.53) are transformed as

$$
\begin{aligned}
& \Psi_{\text {phys }}^{(q)}\left(\begin{array}{c}
c \\
d
\end{array}\right)[\theta, K, \gamma ; t] \rightarrow \exp \left[\frac{2 \pi i}{k} s_{k} M^{k \ell} t_{\ell}+\frac{2 \pi i}{k_{1} M}\left(-s_{k} c^{k}+d_{k} M^{k \ell} t_{\ell}\right)-\frac{2 \pi i}{k_{1} M} s_{k} q^{k}\right] \\
& \times \Psi_{\text {phys }}^{\left(q^{k}-k_{2} M^{k \ell} t_{\ell}\right)}\left(\begin{array}{c}
c \\
d
\end{array}\right)[\theta, K, \gamma ;-\infty] \\
&=\sum_{q^{\prime}}[V(s, t)]_{q q^{\prime}} \Psi_{\text {phys }}^{\left(q^{\prime}\right)}\left(\begin{array}{c}
c \\
d
\end{array}\right)[\theta, K, \gamma ;-\infty]
\end{aligned}
$$

where the matrices

$$
[V(s, t)]_{q q^{\prime}}=\exp \left[\frac{2 \pi i}{k} s_{k} M^{k \ell} t_{\ell}+\frac{2 \pi i}{k_{1} M}\left(-s_{k} c^{k}+d_{k} M^{k \ell} t_{\ell}\right)-\frac{2 \pi i}{k_{1} M} s_{k} q^{k}\right] \delta_{q^{k}-k_{2} M^{k \ell} t_{\ell}, q^{k}}
$$


form a $\left(k_{1}\right)^{p}$ dimensional representation of a variation of a clock algebra

$$
V(s, t) V\left(s^{\prime}, t^{\prime}\right)=\mathrm{e}^{\frac{2 \pi i}{k}\left(s_{k} M^{k \ell} t_{\ell}^{\prime}-s_{k}^{\prime} M^{k \ell} t_{\ell}\right)} V\left(s^{\prime}, t^{\prime}\right) V(s, t)
$$

which can be viewed as the dual of the algebra (4.27) with $k_{1}$ and $k_{2}$ interchanged and the linking matrix $M_{k \ell}$ replaced by its inverse $M^{\ell k}$.

\subsection{Discussion and Conclusions}

We have shown in the above that the full Hilbert space of BF theory with sources is $\left(k_{1} k_{2} M\right)^{p}$ dimensional and the wavefunctions carry a one-dimensional unitary representation of the fractional particle-string exchange holonomies through the angle function $\Phi_{a b}^{(g)}$ in (4.53), with statistics parameter $\frac{k}{2 \pi}$. The wavefunctions in addition carry a 1 dimensional projective representation of the local gauge symmetries, as well as a $\left(k_{2}\right)^{p}$ dimensional projective representation of the algebra (4.27) of large gauge transformations with the 2-cocycle

$$
\alpha_{2}\left(n, m ; n^{\prime}, m^{\prime}\right)=\frac{k_{1} M}{k_{2}}\left(n^{\ell} M_{\ell n} m^{\prime n}-n^{\ell} M_{\ell n} m^{n}\right)
$$

They also carry the corresponding representation of the dual algebra, obtained by interchanging $k_{1}$ and $k_{2}$ and inverting $M_{k \ell}$, where the dual tranformations transport the particle and string sources around the non-trivial homology cycles in $\mathcal{M}_{3}$. What is also interesting is that when the space $\mathcal{M}_{3}$ does contain non-trivial homology cycles, the spectrum of the pure source-free BF theory is also non-trivial and provides quantum representations of the non-trivial cohomology of $\mathcal{M}_{3}$. In all of this, when the linkings of the homology 1-cycles with the homology 2-cycles is non-trivial the linking matrix $M^{\ell m}$ plays an important role: It appears in both the dimensionality of the Hilbert space and the U(1) 2-cocycle that arises in the projective cohomology representations of $\mathcal{M}_{3}$. Its appearence is quite nice, since a complete homological representation should also reflect the non-triviality of $M^{\ell m}$ in these cases.

The above results provide a full, detailed description of the enlarged Hilbert space of the particle-string system, with inner product (4.55), and we see precisely how the fractional linkings of particles and strings are realized in the physical states. The discrete transformation groups above, along with the fractional exchange holonomies, are the analogs of the braid group representations that one obtains in conventional anyon theories [1,24], i.e. 
they represent the generators of the fundamental homotopy group of the particle-string configuration space. For a system of identical, oriented strings this fundamental group is known as the motion group $[15,25]$. In the present case the wavefunctions carry a unitary representation of both the generators of this group which correspond to the exchange holonomies between particles and strings and, in the case of a topologically non-trivial spatial manifold, the generators which are associated with the windings of the sources around the non-trivial homology cycles of the manifold. These latter topological generators are dual to the generators of large gauge transformations, and we see that the BF-theory leads to a duality between large gauge transformations and linking operations. This inherent duality in the wavefunctions yields a quantum representation of the cohomology of the space on which the BF theory is defined, and also of the possible non-trivial linkings of the homology cycles. Thus the BF-type topological field theory description of the adiabatic transports which occur in such a model is well-suited to describe the fractional statistics which can occur in 4 dimensions, and it provides a phenomenological description of anyons propagating in 3-dimensional space, and of the fractional statistics of strings themselves. It also provides interesting quantum representations of the non-trivial topological properties that $\mathcal{M}_{3}$ may possess, and of the resulting motion group on $\mathcal{M}_{3}$.

\section{APPENDIX: ADIABATIC LINKING NUMBERS IN CURVED SPACES}

In this Appendix we present some details of the calculation of the curved space solid angle function which was introduced in (4.50). Many of the calculations in a curved space are performed by decomposing the space of functions on $\mathcal{M}_{3}$ in terms of the eigenfunctions of the scalar Laplacian operator

$$
\nabla_{g}^{2} \psi_{\lambda}(x)=* d * d \psi_{\lambda}(x)=\lambda^{2} \psi_{\lambda}(x)
$$

These functions can be normalized so that

$$
\int_{\mathcal{M}_{3}} \psi_{\lambda} * \psi_{\lambda^{\prime}}=\delta_{\lambda, \lambda^{\prime}}
$$

In the case of degeneracies it is always possible to define an orthonormal set in the degenerate subspace, and this will be implicitly assumed in this Appendix. One important 
function is the constant function $\psi_{0}=\left(\int_{\mathcal{M}_{3}} d^{3} x\right)^{-\frac{1}{2}}$, the single zero mode solution of (A.1) in the space of functions on $\mathcal{M}_{3}$. This decomposition can be used to represent the Dirac delta function on this space through the completeness relation

$$
\delta^{(3)}(x, y)=\sum_{\lambda} \psi_{\lambda}(x) \psi_{\lambda}(y) \quad \text { or } \quad \delta^{(0,3)}(x, y) d^{3} y=\sum_{\lambda} \psi_{\lambda}(x) * \psi_{\lambda}(y)
$$

The above decomposition for functions on $\mathcal{M}_{3}$ is the Hodge decomposition for 0 -forms, the elements of $\Lambda^{0}\left(\mathcal{M}_{3}\right)$. Every 0 -form can be expressed in terms of a coexact 0 -form, $\psi_{\lambda}$ for $\lambda \neq 0$, an exact 0 -form, which doesn't exist since there are no $(-1)$-forms, and a harmonic 0-form, the constant function $\psi_{0}$. A similar decomposition exists for 1-forms on $\mathcal{M}_{3}$, the elements of $\Lambda^{1}\left(\mathcal{M}_{3}\right)$. The exact 1-forms are

$$
\psi_{\lambda}^{(e)}=\frac{d \psi_{\lambda}}{\sqrt{-\lambda^{2}}} \text { with } \int_{\mathcal{M}_{3}} \psi_{\lambda}^{(e)} \wedge * \psi_{\lambda^{\prime}}^{(e)}=\delta_{\lambda, \lambda^{\prime}}
$$

for $\lambda \neq 0$, while the coexact 1 -forms are a new set of forms satisfying

$$
* d * \psi_{\tilde{\lambda}}^{(c)}=0 \quad, \quad\left(\nabla_{g}^{(1)}\right)^{2} \psi_{\tilde{\lambda}}^{(c)}=* d * d \psi_{\tilde{\lambda}}^{(c)}=\tilde{\lambda}^{2} \psi_{\tilde{\lambda}}^{(c)}
$$

which are also normalized as

$$
\int_{\mathcal{M}_{3}} \psi_{\tilde{\lambda}}^{(c)} \wedge * \psi_{\tilde{\lambda}^{\prime}}^{(c)}=\delta_{\tilde{\lambda}, \tilde{\lambda}}
$$

The index $(c)$ will be dropped from here on to simplify notation. We consider only those $\psi_{\tilde{\lambda}}$ with $\tilde{\lambda} \neq 0$, which in this decomposition then leaves only the harmonic 1-forms given by the $\alpha_{\ell}$ introduced at the beginning of Section 4 . They are normalized by the relation $(4.3)$.

It is also possible to define a Dirac delta function for 1-forms in the following sense. Such a function belongs to the space $\Lambda^{1}\left(\mathcal{M}_{3}(x)\right) \otimes \Lambda^{2}\left(\mathcal{M}_{3}(y)\right)$, and when it is wedged with a 1-form $\alpha(y) \in \Lambda^{1}\left(\mathcal{M}_{3}(y)\right)$ and the resulting 3-form on $\Lambda^{3}\left(\mathcal{M}_{3}(y)\right)$ is integrated over $\mathcal{M}_{3}$, we are left with the 1 -form $\alpha(x) \in \Lambda^{1}\left(\mathcal{M}_{3}(x)\right)$. From the Hodge decomposition above, it is rather straightforward to show that this delta function has the representation in terms of the completeness relation

$$
\delta^{(1,2)}(x, y)=-\sum_{\lambda \neq 0} \frac{d \psi_{\lambda}(x) * d \psi_{\lambda}(y)}{\lambda^{2}}+\sum_{\tilde{\lambda}} \psi_{\tilde{\lambda}}(x) * \psi_{\tilde{\lambda}}(y)+\alpha_{\ell}(x) M^{m \ell} \beta_{m}(y)
$$

Note that from (A.7) we can represent the linking matrix (4.1) of $\mathcal{M}_{3}$ as

$$
M^{m n}=\int_{\Sigma^{m}(y)} \int_{L^{n}(x)} \delta^{(1,2)}(x, y)
$$


which is valid only with the normalization (4.2) of the harmonic generators. The Hodge decompositions of 2 -forms and 3 -forms are the Hodge-duals of the above decompositions of 1 -forms and 0 -forms, respectively.

If we look at the definitions of the particle current (2.11) and string current (2.12) and integrate the time components, we recognize the delta functions (A.3) and (A.7), respectively. Using the decomposition (4.39) and (4.40) for the particle current (2.11), we find that

$$
\begin{gathered}
j^{0}(x, t)=\sum_{a} q_{a} \sum_{\lambda} \psi_{\lambda}(x) \psi_{\lambda}\left(r_{a}(t)\right) \\
\Omega(x, t)=\sum_{a} q_{a} \sum_{\tilde{\lambda}} \frac{1}{\tilde{\lambda}^{2}} * d \psi_{\tilde{\lambda}}(x) \frac{\partial}{\partial t}\left(\int_{r_{0}}^{r_{a}(t)} \psi_{\tilde{\lambda}}\right)
\end{gathered}
$$

and

$$
\omega^{\prime}(x, t)=-\sum_{a} q_{a} \sum_{\lambda \neq 0} \frac{1}{\lambda^{2}} \psi_{\lambda}(x) \frac{\partial \psi_{\lambda}\left(r_{a}(t)\right)}{\partial t}=-\frac{1}{\left(\nabla_{g}^{2}\right)^{\prime}} \frac{\partial j^{0}(x, t)}{\partial t}
$$

with the harmonic part

$$
j_{\ell}(t)=\sum_{a} q_{a} \frac{\partial}{\partial t}\left(\int_{r_{0}}^{r_{a}(t)} \alpha_{\ell}\right)
$$

which is just equation (4.41). Here $r_{0}$ is some fixed basepoint in $\mathcal{M}_{3}$.

Similarily from the string current decomposition (4.42) and (4.43) of (2.12) we find

$$
\begin{gathered}
\Sigma_{0}=\Sigma_{0 i} d x^{i}=\sum_{b} \phi_{b} \sum_{\tilde{\lambda}} \psi_{\tilde{\lambda}}(x)\left(\int_{\sigma_{b}(t)} \psi_{\tilde{\lambda}}\right) \\
\pi(x, t)=-\sum_{b} \phi_{b} \sum_{\lambda \neq 0} \frac{1}{\lambda^{2}} \psi_{\lambda}(x) \frac{\partial}{\partial t}\left(\int_{\Sigma_{b}(t)} * d \psi_{\lambda}\right)
\end{gathered}
$$

and

$$
\Pi^{\prime}(x, t)=\sum_{b} \phi_{b} \sum_{\tilde{\lambda}} \frac{1}{\tilde{\lambda}^{2}} \psi_{\tilde{\lambda}}(x) \frac{\partial}{\partial t}\left(\int_{\sigma_{b}(t)} \psi_{\tilde{\lambda}}\right)=\frac{1}{\left(\nabla_{g}^{(1) 2}\right)^{\prime}} \frac{\partial \Sigma_{0 i}(x, t)}{\partial t} d x^{i}
$$

with the harmonic part

$$
\Sigma_{\ell}(t)=\sum_{b} \phi_{b} \frac{\partial}{\partial t}\left(\int_{\Sigma_{b}(t)} \beta_{\ell}\right)
$$

which is equal to (4.44). By $\sigma_{b}(t)$ we mean the string embedding $X_{b}^{i}(t, \sigma)$, while $\Sigma_{b}(t)$ represents a surface, the string world sheet projected onto $\mathcal{M}_{3}$, having the boundary $\sigma_{b}(t)$ at time $t$. 
Let us now compute the expression (4.49) using the relations (A.9), (A.10), (A.13) and (A.14). We find that

$$
\begin{aligned}
\mathcal{L}(t) & =\int_{\mathcal{M}_{3}} d^{3} x\left(\pi(x, t) j^{0}(x, t)-\Omega_{i}(x, t) \Sigma^{0 i}(x, t)\right) \\
& =\sum_{a, b} q_{a} \phi_{b}\left[-\sum_{\lambda \neq 0} \frac{1}{\lambda^{2}} \psi_{\lambda}\left(r_{a}(t)\right) \frac{\partial}{\partial t}\left(\int_{\Sigma_{b}(t)} * d \psi_{\lambda}\right)-\sum_{\tilde{\lambda}}\left(\int_{\Sigma_{b}(t)} * \psi_{\tilde{\lambda}}\right) \frac{\partial}{\partial t}\left(\int_{r_{0}}^{r_{a}(t)} \psi_{\tilde{\lambda}}\right)\right]
\end{aligned}
$$

Integrating the first term in (A.17) by parts over time gives

$$
\begin{aligned}
\mathcal{L}(t)=\sum_{a, b} & q_{a} \phi_{b} \frac{\partial}{\partial t}\left[-\sum_{\lambda \neq 0} \frac{1}{\lambda^{2}} \psi_{\lambda}\left(r_{a}(t)\right)\left(\int_{\Sigma_{b}(t)} * d \psi_{\lambda}\right)\right] \\
& +\sum_{a, b} q_{a} \phi_{b}\left(\alpha_{\ell}\right)_{i}\left(r_{a}(t)\right) \dot{r}_{a}^{i}(t) M^{m \ell}\left(\int_{\Sigma_{b}(t)} \beta_{m}\right) \\
& -\sum_{a, b} q_{a} \phi_{b}\left[-\sum_{\lambda \neq 0} \frac{1}{\lambda^{2}}\left(\int_{\Sigma_{b}(t)} * d \psi_{\lambda}\right) \partial_{i} \psi_{\lambda}\left(r_{a}(t)\right)\right. \\
& \left.+\sum_{\tilde{\lambda}}\left(\int_{\Sigma_{b}(t)} * \psi_{\tilde{\lambda}}\right)\left(\psi_{\tilde{\lambda}}\right)_{i}\left(r_{a}(t)\right)+\left(\int_{\Sigma_{b}(t)} \beta_{m}\right) M^{m \ell}\left(\alpha_{\ell}\right)_{i}\left(r_{a}(t)\right)\right] \dot{r}_{a}^{i}(t)
\end{aligned}
$$

The last 3 terms in (A.18) can be collected together to give the delta function (A.7) integrated over $\Sigma_{b}(t)$, and then comparing the second term in (A.18) with (A.12) and (A.16) we find

$$
\mathcal{L}(t)=-\frac{1}{4 \pi} \sum_{a, b} q_{a} \phi_{b} \frac{d \Phi_{a b}^{(g)}(t)}{d t}+j_{\ell}(t) M^{m \ell} \int_{-\infty}^{t} \Sigma_{m}\left(t^{\prime}\right) d t^{\prime}
$$

where we have defined the function $\Phi_{a b}^{(g)}$ by

$$
\begin{array}{r}
\Phi_{a b}^{(g)}(t)=4 \pi \int_{-\infty}^{t}\left(\int_{\Sigma_{b}\left(t^{\prime}\right)} \delta^{(1,2)}\left(r_{a}\left(t^{\prime}\right), X_{b}\left(t^{\prime}, \sigma\right)\right)\right)_{i} \dot{r}_{a}^{i}\left(t^{\prime}\right) d t^{\prime} \\
+4 \pi \sum_{\lambda \neq 0} \frac{1}{\lambda^{2}} \psi_{\lambda}\left(r_{a}(t)\right)\left(\int_{\Sigma_{b}(t)} * d \psi_{\lambda}\right)
\end{array}
$$

We need to check that $\Phi_{a b}^{(g)}$ is independent of the chosen surface $\Sigma_{b}(t)$ given its boundary $\sigma_{b}(t)$. If we choose a different surface $\tilde{\Sigma}_{b}(t)$ with $\Sigma_{b}-\tilde{\Sigma}_{b}=\partial B_{b}$ for some volume $B_{b}$, 
then the second term in (A.20) will change by

$$
\delta \Phi_{a b}^{(g)}(t)=4 \pi \sum_{\lambda \neq 0} \frac{1}{\lambda^{2}} \psi_{\lambda}\left(r_{a}(t)\right)\left(\int_{\Sigma_{b}(t)-\tilde{\Sigma}_{b}(t)} * d \psi_{\lambda}\right)
$$

Using Stokes' theorem we then find

$$
\delta \Phi_{a b}^{(g)}(t)=4 \pi \sum_{\lambda \neq 0} \int_{B_{b}(t)} \psi_{\lambda}\left(r_{a}(t)\right) \psi_{\lambda}(x) d^{3} x=4 \pi \int_{B_{b}(t)}\left[\delta^{(3)}\left(r_{a}(t), x\right)-\psi_{0} \psi_{0}\right] d^{3} x
$$

This shows that if we continuously deform $\Sigma_{b}(t)$ then the second term in (A.20) does not change unless we cross the particle at $r_{a}(t)$, and then the change is $4 \pi$ which will be cancelled by the first term in (A.20). The contribution from the $\psi_{0}$ function in (A.22) will vanish when we sum over $q_{a}$ and $\phi_{b}$ in (A.19).

A string encircling a fixed particle (i.e. $r_{a}(t)$ constant) once can be represented by $\Sigma_{b}\left(t_{1}\right)=\Sigma_{b}\left(t_{2}\right)$ after it has swept out a closed volume containing the particle in time $t_{2}-t_{1}$, and in this case the second term in (A.20) will add $4 \pi$ to $\Phi_{a b}^{(g)}$. Alternatively, if the string is fixed we can choose $\Sigma_{b}(t)$ constant, and when a particle encircles the string and returns back to its original position the only contribution to $\Phi_{a b}^{(g)}$ is the first term in (A.20) which precisely counts the number of times it crosses $\Sigma_{b}$, giving again the correct linking number. The function (A.20) therefore has the properties that it increases by $4 \pi$ everytime that particle $a$ and string $b$ link themselves exactly once, and furthermore it is independent of the paths of the particles and strings, as long as they do not intersect. (A.20) also reduces to the standard Euclidean space solid angle when the string and particle paths are infinitesimal, since then this function becomes the angle function (3.11). The canonical linking function $\Phi^{(g)}(t)$ above is the adiabatic limit of the covariant linking number

$$
I_{\mathcal{M}_{4}}(\Sigma, L)=\int_{B(\Sigma)} \Delta_{L}
$$

in $\mathcal{M}_{4}$ of a loop $L$ and a closed surface $\Sigma[2,3]$. Here $B(\Sigma)$ is a volume bounded by $\Sigma$ and $\Delta_{L}$ is the deRham current [17] which is the Poincaré-dual of the embedding of $L$ in $\mathcal{M}_{4}$. The deRham current can be written as $\Delta_{L}=\int_{L} \Delta$ where $\Delta$ is the Dirac delta function $\delta^{(1,3)}(r(\tau), x)$ in the space $\Lambda^{1}\left(\mathcal{M}_{4}(r(\tau))\right) \otimes \Lambda^{3}\left(\mathcal{M}_{4}(x)\right)$ which restricts the domain of integration over $\mathcal{M}_{4}$ to $L$, i.e. $\int_{\mathcal{M}_{4}} \Delta_{L} \wedge \alpha=\int_{L} \alpha$ for any 1-form $\alpha$. 


\section{References}

[1] G. W. Semenoff, in Proceedings of the Karpacz Winter School in Theoretical Physics (1993).

[2] D. Birmingham, M. Blau, M. Rakowski and G. Thompson, Phys. Rep. 209 (1991), 129.

[3] M. Blau and G. Thompson, Ann. Phys. 205 (1991), 130.

[4] G. T. Horowitz, Commun. Math. Phys. 125 (1989), 417; M. Blau and G. Thompson, Phys. Lett. B228 (1989), 64; A. Lahiri, Mod. Phys. Lett. A8 (1993), 2403.

[5] G. T. Horowitz and M. Srednicki, Commun. Math. Phys. 130 (1990), 83; I. Oda and S. Yahikozawa, Phys. Lett. B238 (1990), 272; S. Wu, Commun. Math. Phys. 136 (1991), 157.

[6] P. G. de Gennes, Superconductivity of Metals and Alloys, W. A. Benjamin, Inc. (New York) (1966); A. P. Balachandran, V. P. Nair, B.-S. Skagerstam and A. Stern, Phys. Rev. D26 (1982), 1443; T. R. Govindarajan, J. Phys. G8 (1982), L17.

[7] C. P. Burgess and A. Kshirsagar, Nucl. Phys. B324 (1989), 157; J. M. Molera and B. A. Ovrut, Phys. Rev. D40 (1989), 1146; K. Lee: The Dual Formulation of Cosmic Strings and Vortices, CERN preprint CERN-TH-6780/93 (1993).

[8] M. J. Bowick, S. B. Giddings, J. A. Harvey, G. T. Horowitz and A. Strominger, Phys. Rev. Lett. 61 (1988), 2823; T. J. Allen, M. J. Bowick and A. Lahiri, Phys. Lett. B237 (1989), 47; B. A. Campbell, M. J. Duncan, N. Kaloper and K. A. Olive, Phys. Lett. B251 (1990), 34; Nucl. Phys. B351 (1991), 778; S. Coleman, J. Preskill and F. Wilczek, Nucl. Phys. B378 (1992), 175.

[9] M. G. Alford and F. Wilczek, Phys. Rev. Lett. 62 (1989), 1071; L. M. Krauss and F. Wilczek, Phys. Rev. Lett. 62 (1989), 1221; M. G. Alford, J. March-Russel and F. Wilczek, Nucl. Phys. B337 (1990), 695; J. Preskill and L. M. Krauss, Nucl. Phys. B341 (1990), 50.

[10] M. I. Polikarpov, U.-J. Wiese and M. A. Zubkov: String Representation of the Abelian Higgs Theory and Aharanov-Bohm Effect on the Lattice, Institute of Theoretical and Experimental Physics preprint ITEP-16-1993 (1993).

[11] A. S. Schwarz, Commun. Math. Phys. 67 (1979), 1; J. Gegenberg and G. Kunstatter, Phys. Lett. B321 (1994), 193.

[12] X. Fustero, R. Gambini and A. Trias, Phys. Rev. Lett. 62 (1989), 1964; J. A. Harvey and J. Liu, Phys. Lett. B240 (1990), 369.

[13] E. Witten, Phys. Lett. B149 (1984), 351.

[14] M. Kalb and P. Ramond, Phys. Rev. D9 (1974), 2273; R. Rohm and E. Witten, Ann. Phys. 170 (1986), 454.

[15] C. Aneziris, A. P. Balachandran, L. H. Kauffman and A. M. Srivastava, Int. J. Mod. Phys. A6 (1991), 2519.

[16] A. P. Balachandran, G. Bimonte and P. Teotonio-Sobrinho, Mod. Phys. Lett. A8 (1993), 1305; A. P. Balachandran and P. Teotonio-Sobrinho, Int. J. Mod. Phys. A8 (1993), 723; A9 (1994), 1569. 
[17] R. Bott and L. W. Tu, Differential Forms in Algebraic Topology, Springer-Verlag (New York) (1986).

[18] D. B. Ray and I. M. Singer, Adv. Math. 7 (1971), 145.

[19] H. Flanders, Differential Forms with Applications to the Physical Sciences, Dover Publications (New York) (1989).

[20] O. Alvarez, Commun. Math. Phys. 100 (1985), 279; A. P. Polychronakos, Nucl. Phys. B281 (1987), 241.

[21] E. Witten, Commun. Math. Phys. 121 (1989), 351.

[22] D. Mumford, Tata Lectures on Theta, Birkhauser (Basel) (1983).

[23] A. M. Perelomov, Generalized Coherent States and Their Applications, Springer-Verlag (Berlin) (1986).

[24] M. Bergeron and G. W. Semenoff, Ann. Phys. (1994), in press.

[25] D. L. Goldsmith, Mich. Math. J. 28 (1981), 3. 